\title{
Análisis de los Grosores Tisulares a Nivel de Nasion y Glabela según Sexo y Biotipo, en Pacientes entre 20 y 27 Años de Edad
}

\author{
Nasion and Glabela Thickness Tissue According to Sex \\ and Biotypein Patients between 20 and 27 Years of Age
}

David Reininger*; Paola Lillo** \& Pedro Solé****

\begin{abstract}
REININGER, D.; LILLO, P. \& SOLÉ, P. Análisis de los grosores tisulares a nivel de nasion y glabela según sexo y biotipo, en pacientes de edad entre 20 y 27 años. Int. J. Morphol., 31(3):963-966, 2013.

RESUMEN: La población chilena se caracteriza por ser una población muy heterogenea, debido a la gran mezcla exístente entre las distintas etnias y razas a lo largo del tiempo. Esto supone una gran diversidad de formas, tamaños y grosores de estructuras anatómicas, dentro de las cuales ubicamos a los grosores faciales. Si bien existen bases de datos de los grosores tisulares faciales en la población chilena, éstas han sido realizadas a través de técnicas como la ultrasonografía y punción, sin existir estudios realizados en Chile utilizando técnicas radiológicas para medir, como es la telerradiografía de perfil. Los objetivos fueron: 1. Medir los grosores tisulares a nivel de GB y NA, 2. Evaluar correlación de grosores según distribución por sexo y biotipo, 3. Comparar grosores entre distintos grupos, 4. Creación de una base de datos de referencia de la población chilena sobre el espesor del tejido blando a nivel de GB y Na y 5. Comparar los resultados obtenidos con otros estudios de grosores realizados en la población chilena. Se escogió al azar a 99 alumnos de odontología entre 20 a 27 años que necesitaban tratamiento de ortodoncia, a los cuales se les solicita una telerradiografía de perfil, en donde se mide los grosores de tejidos blandos a nivel de glabela y nasion, se clasifican a los pacientes según sexo y biotipo utilizando el análisis de Roth-Jaraback. Al analizar los resultados según sexo se observó siempre un grosor mayor en hombre que en mujer, con diferencia significativa a nivel de glabela y nasion. Al evaluar los resultados según biotipo no se observa diferencias siginificativas entre un biotipo y otro. Los hombres presentan un mayor grosor de tejido blando a nivel de glabela y nasion, con diferencia significativa en ambos. Se propone una nueva clasificación según biotipo, pero no se encuentra resultados concluyentes.
\end{abstract}

PALABRAS CLAVE: Tejidos blandos faciales; Reconstrucción facial; Resonancia nuclear magnetica; Tomografía computada; Telerradiografía de perfil.

\section{INTRODUCCIÓN}

La población chilena se caracteriza por ser una población muy heterogenea, existiendo una gran mezcla entre etnias, razas de distintas zonas del mundo, siendo muy difícil de encontrar rasgos característicos propios del chileno.

Al presentar dicha característica se tendería a pensar la disparidad de formas, tamaños, grosores de estructuras faciales, presentando gran variedad de rasgos físicos, siendo muy complicado el poder encontrar un patrón común. Fenómeno que nos lleva a analizar algunos rasgos anatómicos con el fin de poder encontrar características en común.

Los rasgos anatómicos a analizar son los grosores tisulares a nivel de Glabela (GB) y Nasion (Na), grosores que han sido estudiados en diversas poblaciones del mundo, siendo muy escasos los estudios en relación a este tema en Chile.
El estudio de los grosores se puede realizar a través de: 1. Punción con aguja (Codinha, 2009; De Greef et al., 2006; Domaracki \& Stephan, 2006): estudio realizado en cadáver, que consiste en introducir una aguja hasta llegar a tejido óseo, luego se determina la medida midiendo la zona de la aguja que fue enterrada. Esta medida presenta cierta distorsión debido a la deshidratación que sufre el cadáver, el grado de distorsión va a depender del tiempo transcurrido entre la muerte y la medición. Se recomienda realizar las mediciones antes de las primeras 24 horas post mortem, y sin haber sido realizada la autopsia. 2. Telerradiografia de perfil (De Greef et al.; Sahni et al., 2008): estudio radiográfico que se caracteriza por ser de bajo costo y presentar bajos índices de radiación. Dentro de sus inconvenientes encontramos que solo nos permite medir estructuras ubicadas en la línea media. 3. Resonancia Magnética (De Greef et al.; Rocha et al., 2003; Sahni

\footnotetext{
Becado Magister Cirugía e Implantología Oral, Universidad Complutense de Madrid, Madrid, España. Investigador Asociado de Cirugía Oral y Maxilofacial, Universidad de Los Andes, Santiago, Chile.

** Cirujano Dentista, Investigador.

*** CMF, Universidad de Los Andes, Santiago, Chile.
} 
et al.): estudio imageneológico en el cual no hay radiación, da una imagen de tejidos blandos ideal para la toma de mediciones, pero presenta el inconveniente de su alto costo. 4. Tomografía Computada (De Greef et al.; Phillips \& Smuts, 1996; Sahni et al.): estudio de gran calidad para evaluar grosores tanto de tejido blando como de tejido duro, se encuentra ampliamente distribuido, tiene el inconveniente de presentar una alta radiación comparada con la telerradiografía de perfil, además de su alto costo. 5. Ultrasonografía (Suazo et al., 2007a): método económico, rápido, no invasivo, que entrega una excelente imagen, siendo su principal limitante la necesidad de un operador entrenado.

El examen utilizado en este estudio para medir los grosores es la telerradiografía de perfil, teniendo como objetivos: 1. Medir los grosores tisulares a nivel de GB y NA, 2. Evaluar correlación de grosores según distribución por sexo y biotipo, 3. Comparar grosores entre distintos grupos, 4. Creación de una base de datos de referencia del espesor del tejido blando a nivel de GB y Na de la población chilena y 5 . Comparar los resultados obtenidos con los otros estudios de grosores realizados en la población chilena.

\section{MATERIAL Y MÉTODO}

Estudio exploratorio de 99 pacientes elegidos al azar, entre estudiantes de odontología de la Universidad de los Andes en Santiago de Chile. Se consideró como criterio de inclusión la edad, la cual debe ser entre 20 y 27 años y la necesidad de realizarse tratamiento de ortodoncia. Fueron excluidos del estudio aquellos que presentaron fracturas nasales, fracturas de seno frontal, malformaciones faciales y tumores faciales.

A todos se le realizó una telerradiografía de perfil, utilizando el equipo radiográfico Sirona, modelo: Orthophos xp plus, ubicando el posicionar del nasion como punto de referencia, tomando las medidas necesarias para no ejercer pre- sión en la zona y así no afectar los resultados de la medición.

Una vez obtenida la telerradiografía se procedió a través del programa Sidexis a marcar los puntos nasion y glabela de tejido blando, midiendo el grosor de la estructura. Se analizaron los resultados generales del grupo evaluando los grosores máximos, mínimos y el promedio. Luego se clasificaron los pacientes según sexo y biotipo (utilizando el análisis de Roth-Jarabak para su clasificación).

El estudio estadístico se realizó con el programa Systat, utilizando los análisis de: t-test pareado y no pareado, Anova y Tukey. Se contó con el consentimiento y aprobación previa de los pacientes, y bajo una evaluación del comité de ética de la misma Universidad de los Andes, la cual aprobó y autorizó la investigación.

\section{RESULTADOS}

En la Tabla I se observan los datos generales obtenidos. El número de paciente distribuido por sexo correspondió a 31 hombres y 68 mujeres, mientras que según biotipo 34 sujetos fueron mesofaciales, 20 dolicofaciales y 45 braquifaciales. Luego de esto se procedió a realizar el análisis según sexo y biotipo.

$\mathrm{Al}$ analizar estadísticamente los resultados de los grosores según sexo (Tabla II), todos los promedios de los grosores son mayores en hombres que en mujeres. Al comparar los grosores se utilizó el análisis de t-test no pareado, encontrándose diferencias significativas en el grosor de glabela $(\mathrm{P}=0,62)$ y de nasion $(\mathrm{P}=0,00)$.

En el análisis estadístico de los grosores según biotipo (Tabla II), para comparar los grosores se utilizaron las pruebas estadísticas de Anova y luego la de Tukey, no se observándose diferencias significativas.

Tabla I. Descripción general de los datos obtenidos.

\begin{tabular}{lcccc}
\hline Variable & Media & DE & Mínima & Máxima \\
\hline Grosor tejido blando glabela & $5,14 \mathrm{~mm}$ & $\pm 0,81 \mathrm{~mm}$ & $3,38 \mathrm{~mm}$ & $7,69 \mathrm{~mm}$ \\
Grosor tejido blando nasion & $5,53 \mathrm{~mm}$ & $\pm 1,12 \mathrm{~mm}$ & $3,28 \mathrm{~mm}$ & $8,24 \mathrm{~mm}$ \\
\hline
\end{tabular}

Tabla II. Resultados obtenidos de los promedios de los grosores en pacientes agrupados por sexo y biotipo.

\begin{tabular}{lcc}
\hline & Promedio grosor GB blando & Promedio grosor Na blando \\
\hline Sexo Femenino & $4,88 \pm 0,62 \mathrm{~mm}$ & $5,19 \pm 0,9 \mathrm{~mm}$ \\
Sexo Masculino & $5,67 \pm 0,91 \mathrm{~mm}$ & $6,29 \pm 6,29 \mathrm{~mm}$ \\
Biotipo Mesofacial & $5,24 \pm 0,92 \mathrm{~mm}$ & $5,41 \pm 1,14 \mathrm{~mm}$ \\
Biotipo Dolicofacial & $4,83 \pm 0,62 \mathrm{~mm}$ & $5,22 \pm 0,97 \mathrm{~mm}$ \\
Biotipo Braquifacial & $5,19 \pm 0,78 \mathrm{~mm}$ & $5,77 \pm 1,15 \mathrm{~mm}$ \\
\hline
\end{tabular}




\section{DISCUSIÓN}

A pesar de estar descrito el uso de la telerradiografía de perfil para medir grosores de tejidos a nivel de la línea media, los estudios encontrados en relación al tema utilizan otro tipo de técnica; ya sea con aguja, uso de TC, RNM o ultrasonografía. En este estudio se eligió la telerradiografía de perfil para realizar las mediciones ya que son exámenes de rigor previo tratamiento ortodóncico, lo que no provocaba para el paciente un mayor gasto, ni una radiación innecesariamente, consiguiendo a través de éste lograr medir adecuadamente los grosores de tejido blando a nivel de GB como de Na.

La mayoría de los estudios encontrados son análisis de mediciones de grosores tisulares faciales en cadáveres, medición que se realizó con la técnica con aguja (Barriga et al., 2010; Codhina; Phillips \& Smuts; Suazo et al., 2007b; Tedeschi-Oliveira et al., 2009). Un estudio se hizo sobre pacientes vivos realizando la medición en resonancia nuclear magnética (Sipahiouglu et al., 2012), otro estudio se realizó midiendo los grosores con tomografía computada en pacientes vivos (Panenková et al., 2012), y un último estudio se realizó utilizando la ultrasonografía para medir los grosores (Suazo et al., 2007a)

$\mathrm{Al}$ analizar y comparar el grosor de GB agrupados por sexo entre los distintos estudios, se observa la tendencia que los hombres presenten un mayor grosor al ser comparados con las mujeres (Barriga et al.; Codhina; Dong et al., 2012; Panenková et al.; Sipahiouglu et al.; Suazo et al., 2007a, 2007b; Tedeschi-Oliveira et al.) (Tablas III), siendo el estudio de la Tedeschi-Oliveira et al., el único que muestra diferencia significativa a este nivel. De igual manera en nuestro estudio los grosores son siempre mayores en hombres que en mujeres (Tabla II), y concordamos con la existencia de diferencia significativa a nivel de GB al comparar los grosores entre sexo masculino y femenino.
Analizando el grosor a nivel de Na, también se observa la tendencia que los hombres presenten mayor grosor al ser comparados con las mujeres (Barriga et al.; Codhina; Dong et al.; Sipahiouglu et al.; Suazo et al., 2007a, 2007b; Tedeschi-Oliveira et al.) (Tablas III), en este caso encontramos múltiples estudios que señalan la existencia de diferencia significativa entre ambos sexos (Barriga et al.; Codhina; Dong et al.; Sipahiouglu et al.; Suazo et al., 2007a, 2007b; Tedeschi-Oliveira et al.). Este estudio concuerda plenamente con los estudios señalados anteriormente, encontrando en todos los casos diferencia significativa (Tabla II).

Respecto a la clasificación según biotipo, se intentó incorporar nuevas variables que pudieran influir en los grosores faciales. Es así como se observó la relación existente entre los distintos biotipos faciales y el grosor de tejidos blandos tanto a nivel de GB como de $\mathrm{Na}$, al evaluar los resultados no se encontró una medida fija estable al analizar en los distintos grosores de los biotipos, pero sí se observa cierta homogeneidad en las medidas faciales de los distintos biotipos. A nivel de glabela el biotipo mesofacial es el que presenta un mayor grosor, seguido por los biotipos braquifacial y dolicofacial, sin existir diferencias significativas entre ellos. En cuanto al grosor en $\mathrm{Na}$ el biotipo braquifacial es el que presenta mayor grosor seguido por los biotipos mesofacial y dolicofacial, sin presentar diferencias significativas (Tabla II).

Otros factores que influyen en el grosor de tejidos blandos a nivel de GB y Na es el peso y la edad (Barriga et al.; Codhina; Dong et al.; Sipahiouglu et al.; Suazo et al., 2007a, 2007b; Tedeschi-Oliveira et al.), siendo mayor en aquellas personas que presenten sobrepeso y mayor edad.

En conclusión,este trabajo pretende aportar información sobre rasgos anatómicos que hasta hoy en día no habían sido estudiados acabadamente a través de técnicas radiológicas en Chile, con el fin de lograr una base de datos de referencia del espesor de tejidos blandos a nivel de GB y Na en una población de edad entre los 20 a 27 años.

Tabla III. Medida de los tejidos blandos en hombres y mujeres en diferentes poblaciones.

\begin{tabular}{lcccc}
\hline & \multicolumn{2}{c}{ Hombres } & \multicolumn{2}{c}{ Mujeres } \\
\cline { 2 - 5 } & Nasion & Glabela & Nasion & Glabela \\
\hline Suazo et al. (2007a) & $5,9 \mathrm{~mm}$ & $5,5 \mathrm{~mm}$ & $5,6 \mathrm{~mm}$ & $5,5 \mathrm{~mm}$ \\
Suazo et al. (2007b) & $5,6 \mathrm{~mm}$ & $4,8 \mathrm{~mm}$ & $5,1 \mathrm{~mm}$ & $4,7 \mathrm{~mm}$ \\
Codhina (2009) & $5,3 \mathrm{~mm}$ & $4,9 \mathrm{~mm}$ & $4,9 \mathrm{~mm}$ & $4,6 \mathrm{~mm}$ \\
Tedesc hi-Oliveir a et al. (2009) & $5,9 \mathrm{~mm}$ & $5,5 \mathrm{~mm}$ & $5,0 \mathrm{~mm}$ & $4,6 \mathrm{~mm}$ \\
Barriga et al. $(2010)$ & $5,2 \mathrm{~mm}$ & $4,68 \mathrm{~mm}$ & $5,2 \mathrm{~mm}$ & $4,68 \mathrm{~mm}$ \\
Sipahiouglu et al. $(2012)$ & $7,34 \mathrm{~mm}$ & $6,28 \mathrm{~mm}$ & $4,5 \mathrm{~mm}$ & $4,61 \mathrm{~mm}$ \\
Panencová et al. $(2012)$ & $7,5 \mathrm{~mm}$ & $5,3 \mathrm{~mm}$ & $7,14 \mathrm{~mm}$ & $6,01 \mathrm{~mm}$ \\
Dong et al. (2012) & $5,98 \mathrm{~mm}$ & $4,59 \mathrm{~mm}$ & $5,6 \mathrm{~mm}$ & $4,5 \mathrm{~mm}$ \\
\hline
\end{tabular}


Los resultados obtenidos en éste estudio son similares a los artículos analizados, concluyéndose: 1 . Siempre son mayores los grosores en hombres que en mujeres, 2. Existe diferencia significativa a nivel de Na, y 3. Según los resultados obtenidos existe diferencia significativa a nivel de GB, resultado similar obtenido por el estudio realizado Tedeschi-Oliveira et al.

REININGER, D.; LILLO, P. \& SOLÉ, P. Nasion and glabela thickness tissue according to sex and biotype in patients between 20 and 27 years of age. Int. J. Morphol., 31(3):963-966, 2013.

SUMMARY: Chilean population is characterized for being heterogeneous, due to a large variety and mix of different ethnicities and races. This includes diversity in shapes, sizes and thickness of anatomic structures, part of which is facial thickness. Even though there are databases of facial tissue thickness for Chilean population, these have been carried out through techniques such as ultrasonography and puncture, without taking into consideration radiologic techniques, such as profile teleradiography. The aims of the study were: 1. Measure tissue thickness at GB and NA level, 2. Evaluate correlation in thickness according to distribution per sex and biotype. 3. Compare thickness among the groups. 4. Create a database of soft tissue thickness at GB and NA level in Chilean population, 5. Compare results with other studies regarding tissue thickness in Chilean population. Ninety-nine dentistry students between 20 and 27 years of age, candidates for orthodontic treatment, were randomly chosen. Lateral teleradiography was requested from all individuals, soft tissue thickness was measured at GB and NA level, classifying patients according to Roth-Jaraback analysis. When analysing the results according to sex, larger thickness was observed in men each time, with a significant difference at GB and NA levels. When evaluating biotype differences no significant difference was observed. Men showed thicker soft tissue at NA and GB level than women, with a significant difference between both. A new classification is proposed according to biotype, but no concluding data is found.

KEY WORDS: Facial soft tissue; Facial reconstruction; Magnetic nuclear resonance; Computed tomography; Lateral telerradiography.

\section{REFERENCIAS BIBLIOGRÁFICAS}

Barriga, C; Zavando, D; Cantín, M. \& Suazo, I. Facial tissue thickness in Chilean cadavers with medico-legal purposes. Int. J. Odontostomat., 4(3):215-22, 2010.

Codinha, S. Facial soft tissue thicknesses for the Portuguese adult population. Forensic Sci. Int., 184(1-3):80.e1-7, 2009.

De Greef, S.; Claes, P.; Vandermeulen, D.; Mollemans, W.; Suetens, P. \& Willems, G. Large-scale in-vivo Caucasian facial soft tissue thickness database for craniofacial reconstruction. Forensic Sci. Int., 159 Suppl 1:S126-46, 2006.

Domaracki, M. \& Stephan, C. N. Facial soft tissue thicknesses in Australian adult cadavers. J. Forensic Sci., 51(1):5-10, 2006.

Dong, Y.; Huang, L.; Feng, Z.; Bai, S.; Wu, G. \& Zhao, Y. Influence of sex and body mass index on facial soft tissue thickness measurements of the northern Chinese adult population. Forensic Sci. Int., 222(1-3):396.e1-7, 2012.

Panenková, P.; Benus, R.; Masnicová, S.; Obertová, Z. \& Grunt, J. Facial soft tissue thicknesses of the mid-face for Slovak population. Forensic Sci. Int., 220(1-3):293.e1-6, 2012.

Phillips, V. M. \& Smuts, N. A. Facial reconstruction: utilization of computerized tomography to measure facial tissue thickness in a mixed racial population. Forensic Sci. Int., 83(1):51-9, 1996.

Rocha, S. S.; Ramos, D. L. \& Cavalcanti, M. G. Applicability of 3D-CT facial reconstruction for forensic individual identification. Pesqui. Odontol. Bras., 17(1):24-8, 2003.
Sahni, D.; Sanjeev; Singh, G.; Jit, I. \& Singh, P. Facial soft tissue thickness in northwest Indian adults. Forensic Sci. Int., 176(2-3):137-46, 2008.

Sipahioglu, S.; Ulubay, H. \& Diren, H. B. Midline facial soft tissue thickness database of Turkish population: MRI study. Forensic Sci. Int., 219(13):282.e1-8, 2012.

Suazo, G. I. C.; Salgado, A. G. E. \& Cantín, L. M. G. Evaluación ultrasonográfica del tejido blando facial en adultos chilenos. Int. J. Morphol., 25(3):643-8, 2007a.

Suazo, G. I. C.; Perez, R. F. J. \& Torres, M. S. R. Grosores tisulares faciales en cadáveres de Españoles y su aplicación en la identificación médicolegal. Int. J. Morphol., 25(1):109-16, 2007b.

Tedeschi-Oliveira, S. V.; Melani, R. F.; de Almeida, N. H. \& de Paiva, L. A. Facial soft tissue thickness of Brazilian adults. Forensic Sci. Int., 193(13):127.e1-7, 2009 .

\author{
Dirección para Correspondencia: \\ Dr. David Reininger \\ Becado Magister Cirugía e Implantología Oral \\ Universidad Complutense de Madrid \\ Madrid \\ ESPAÑA
}

Email: d.reininger@gmail.com

Recibido : 17-01-2013

Aceptado: 13-04-2013 